\title{
Molecular identification of medicinal herbs, Oldenlandia diffusa and Oldenlandia corymbosa based on nrDNA ITS region sequence
}

\author{
Yan-Lin Sun · Dong Wang $\cdot$ Myung-Hun Yeom $\cdot$ Duck-Hee Kim $\cdot$ Han-Gon Kim $\cdot$ Soon-Kwan Hong
}

Received: 19 August 2011 / Accepted: 5 September 2011

(c) Korean Society for Plant Biotechnology

\begin{abstract}
The medicinal herb Oldenlandia diffusa is known as a folk medicine for the treatment of hepatitis, sore throat, appendicitis, malignant tumors and urethral infection in Southern China and Korea. Another species O. corymbosa, is also used for the therapy of the similar conditions, however, only $O$. diffusa is referred to the medicinal herb by Chinese Pharmacopoeia. Due to their similar morphology, $O$. diffusa and $O$. corymbosa are often misidentified. To easily identify $O$. diffusa from $O$. corymbosa, the phylogenetic utility of nuclear ribosomal DNA (nrDNA) internal transcribed spacers (ITS) were investigated among different $O$. diffusa and $O$. corymbosa populations in Korea. The nrDNA ITS sequence of $O$. diffusa contained $791 \mathrm{bp}$, with GenBank accession number of JF837601-JF837602. The nrDNA ITS sequence of $O$. corymbosa was $785-786 \mathrm{bp}$, with GenBank accession number of JF837603-JF837611. The results showed that there are some certain divergences in the ITS region sequence between both species, even among different populations of the same species. Particularly, $O$. corymbosa ST-4 population showed the highest dissimilarity of the ITS region sequence with other nine populations of $O$. corymbosa and two populations of $O$. diffusa. This consequence makes us further understand the molecular diversification between $O$. corymbosa and $O$. diffusa, and help to
\end{abstract}

Y.-L. Sun

School of Life Sciences, Ludong University, Yantai, 264-025, China

Y.-L. Sun $\cdot$ D. Wang $\cdot$ S.-K. Hong $(\bowtie)$

Department of Bio-Health Technology, College of Biomedical Science, Kangwon National University, Chuncheon,

Kangwon-Do, 200-701, Korea

e-mail: soonkwan@kangwon.ac.kr

M.-H. Yeom • D.-H. Kim • H.-G. Kim

R\&D Center, Amore Pacific Co. Yongin, 446-929, Korea

S.-K. Hong

Institute of Bioscience and Biotechnology, Kangwon National

University, Chuncheon, Kangwon-Do, 200-701, Korea promote the correct use and safety.

Keywords Oldenlandia diffusa, Oldenlandia corymbosa, internal transcribed spacer, ribosomal RNA gene, molecular analysis

\section{Introduction}

The herb of Oldenlandia diffusa (also known by alternate Hedyotis diffusa) is known as a traditional, folk medicine in Southern China (Liang et al. 2008). It is traditionally used as an herbal tea component, and for the treatment of sore throat, infectious diseases and malignant tumors (Shan et al. 2001; Kitani et al. 2011; Wu et al. 2009; Xu et al. 1997). Another species, O. corymbos (also known by alternate Hedyotis corymbosa) belonging to the same genus with $O$. diffusa, is also used in modern Chinese practice for the treatment of viral infections, cancer, some syndromes involving toxic heat, acne, boils, skin ailments, appendicitis, hepatitis, eye diseases and bleeding (Corbineau et al. 1980; Lin et al. 1987). Due to their similar morphological characters (Zhao and Li 2007), both $O$. diffusa and $O$. corymbosa are frequently confused with each other in the wholesale and food markets (Liang et al. 2006; Li et al. 2010). However, according to the Chinese Pharmacopoeia (The State Pharmacopoeia Committee of China 2005), the medical herb refers only to the dried herb of $O$. diffusa (Liang et al. 2006). The misusing has attracted an increasing attention on their use security. To make sure of their secure uses, clearer classification between these both medicinal herbs is urgently needed.

Many investigations have been reported to attempt to distinguish $O$. diffusa from $O$. corymbosa using various classification methods such as fluorescence microscopy (Lau et al. 2004), morphological analysis (Ko 1999; Liang et al. 2006), chemical fingerprints (Liang et al. 2008) and DNA 
sequence analysis (Hao et al. 2004; Liu et al. 2004; Guo et al. 2011). Particularly, Liang et al. (2006) reported that the two species could be distinguished with each other according to transverse sections of stems; Liu et al. (2004) newly designed $O$. diffusa-specific PCR primers for internal transcribed spacer 2 (ITS2) region amplification to successfully identify $O$. diffusa from adulterated $O$. corymbosa; Guo et al. (2011) investigated five DNA barcoding regions including ITS, matK, petD, trnH-psbA and $r b c L$ among 25 Oldenlandia species and constructed a phylogenetic tree. Nowadays, simple conventional morphological methodology for taxon classification has not satisfied the increasing demands of more refined identification. Compared to the conventional identification methods, molecular identification based on DNA cloning and sequencing has been considered a more efficient, faster means. By more rapidly providing more phylogenetic information, molecular marker identification method has been more frequently utilized for phylogenetic analysis. Among various molecular identification methods, the sequence analysis of ITS, a region of ribosomal RNA is considered as an effective method to give a higher number of informative sites at species levels (Kitani et al. 2009; Long et al. 2004).

In this study, we investigated the 18S-26S nuclear ribosomal DNA (nrDNA) ITS1 and ITS2 sequence variations between $O$. diffusa and $O$. corymbosa, to distinguish these two similar species in the molecular levels. This work would provide more ITS sequence information on $O$. diffusa and $O$. corymbosa species, help further understand the nucleotide variations among different Korea populations of $O$. diffusa and
$O$. corymbosa, and then identify the identification efficiency based on ITS region sequence between the both species.

\section{Materials and Methods}

Plant materials

Nine populations of $O$. corymbosa and two populations of $O$. diffusa collected from Korea, were used in this study. The populations were named as ST-L, ST-S, ST-1, ST-2, ST-3, ST-4, ST-5, ST-6 and ST-7 of $O$. corymbosa, and LT-L and LT-S of $O$. diffusa, for the convenience of sample management. Mature seeds of $O$. diffusa and $O$. corymbosa were provided by Research and Development Center of Amore-Pacific Co., 446-729, Yongin, Korea. The mature seeds of both species were surface-sterilized with $70 \%$ ethanol for 30 seconds and then rinsed with sterile water for five times. The sterilized seeds were then grown in a pot containing a mixture of sterilized soil and vermiculite (v:v, 3:1) in a green house condition with $40 \sim$ $60 \%$ relative wet rate, a 16/8 h (light/dark) photoperiod, and $24 \pm 2{ }^{\circ} \mathrm{C}$. After germination, the fresh leaf tissue was sampled and used for DNA extraction. The total or partial ITS sequences of $O$. corymbosa and $O$. diffusa have been published by GenBank, NCBI, with the accession numbers shown in Table 1.

DNA extraction and PCR amplification

DNA was extracted from fresh leaves of $O$. diffusa and $O$.

Table 1 ITS region sequence information about GenBank accession number, the length (bp) and (G+C) \% of ITS1, 5.8S and ITS2 of 9 populations of $O$. corymbosa and 2 populations of $O$. diffusa

\begin{tabular}{lccccccccccc}
\hline \multirow{2}{*}{ Species } & Population & $\begin{array}{c}\text { Accession } \\
\text { number }\end{array}$ & $\begin{array}{c}\text { ITS1 } \\
\text { integrity } \\
\text { degree }\end{array}$ & $\begin{array}{c}\text { Length } \\
(\mathrm{bp})\end{array}$ & $\begin{array}{c}\text { ITS1 } \\
(\mathrm{G}+\mathrm{C}) \%\end{array} \%$ & $\begin{array}{c}\text { ITS2 } \\
\text { integrity } \\
\text { degree }\end{array}$ & $\begin{array}{c}\text { ITS2 } \\
\text { Length } \\
(\mathrm{bp})\end{array}$ & $\begin{array}{c}\text { ITS2 }(\mathrm{G}+\mathrm{C}) \\
\%\end{array}$ & $\begin{array}{c}5.8 \mathrm{~S} \\
\text { integrity } \\
\text { degree }\end{array}$ & $\begin{array}{c}5.8 \mathrm{~S} \\
\text { Length } \\
(\mathrm{bp})\end{array}$ & $\begin{array}{c}5.8 \mathrm{~S}(\mathrm{G}+\mathrm{C}) \\
\%\end{array}$ \\
\hline \multirow{2}{*}{ O. diffusa } & LT-L & JF837601 & + & 328 & 54.27 & + & 300 & 60.00 & + & 163 & 53.37 \\
& LT-S & JF837602 & - & 232 & 58.62 & - & 263 & 61.22 & + & 163 & 53.37 \\
\hline & ST-L & JF837603 & + & 318 & 55.66 & + & 304 & 60.20 & + & 163 & 53.37 \\
& ST-S & JF837611 & - & 233 & 58.80 & - & 268 & 61.19 & + & 163 & 53.37 \\
& ST-1 & JF837605 & - & 232 & 59.05 & - & 279 & 59.86 & + & 163 & 53.37 \\
& ST-2 & JF837606 & - & 233 & 57.94 & - & 263 & 61.60 & + & 163 & 53.37 \\
& ST-3 & JF837607 & - & 226 & 59.29 & - & 279 & 60.57 & + & 163 & 53.37 \\
& ST-4 & JF837608 & - & 152 & 62.50 & - & 259 & 58.69 & + & 163 & 53.37 \\
& ST-5 & JF837609 & - & 232 & 59.05 & - & 268 & 61.19 & + & 163 & 53.37 \\
& ST-6 & JF837610 & - & 234 & 58.97 & - & 279 & 59.86 & + & 163 & 53.37 \\
& ST-7 & JF837604 & + & 322 & 51.86 & + & 301 & 60.80 & + & 163 & 53.37 \\
\hline
\end{tabular}

+ presents complete sequence, - presents partial sequence 
corymbosa using the modified cetyltrimethylammonium bromide (CTAB) method described by Kang et al. (1998). Common ITS primer sets ITS5, 5'-GAA AGT AAA AGT CGT AAC AAG G-3' and ITS4, 5'- TCC TCC GCT TAT TGA TAT GC-3' were used to amplify the nrDNA ITS region including ITS1, 5.8S rRNA, ITS2 regions (White et al. 1990). PCR amplification was conducted using this set of primers with the following program: 35 cycles of denaturation at $95^{\circ} \mathrm{C}$ for $1 \mathrm{~min}$, annealing at $53^{\circ} \mathrm{C}$ for $1 \mathrm{~min}$, and a final extension step at $72^{\circ} \mathrm{C}$ for $1.5 \mathrm{~min}$. All PCR products were purified before DNA sequence analysis using a QIAquick PCR Purification Kit (QIAGEN, Korea) according to the manufacturer's instructions. Purified PCR products were then sequenced at MACRO GEN Advancing through Genomics (Korea).

Sequence analysis

Homology analysis of the 11 ITS region sequences were performed by DNAMAN 6.0 software according to the Observed Divergency distance method. The phylogenetic tree of $O$. corymbosa and $O$. diffusa were also constructed based on neighbor joining method using DNAMAN 6.0.

\section{Results and Discussion}

Length and $\mathrm{G}+\mathrm{C}$ content

Total ITS region sequences were successfully amplified by PCR using common primer sets, ITS4/ITS5 from DNAs of nine $O$. corymbosa populations and two $O$. diffusa populations. Due to inefficient reading with nucleotide overlapping appeared in sequencing results, only three total ITS region sequences were obtained from one $O$. diffusa population, LT-L and two O. corymbosa populations, ST-L and ST-7 (Table 1). According to the total ITS region sequences of $O$. diffusa LT-L population, the size of the total ITS region was $791 \mathrm{bp}$, of which 328 bp belonged to ITS1 region, 163 bp belonged to 5.8S rRNA gene, and $300 \mathrm{bp}$ belonged to ITS2 region. For $O$. corymbosa, the total ITS region accounted for 785 or 786 bp varying in ST-L or ST-7 population, respectively, of which ITS1 region accounted for $318 \sim 322 \mathrm{bp}$, ITS2 region for $301 \sim 304 \mathrm{bp}$, and 5.8S rRNA region for $163 \mathrm{bp}$ with the identical size of $O$. diffusa (Table 1). Not only sequence lengths but $(\mathrm{G}+\mathrm{C})$ contents (\%) of ITS region sequences were different among different populations of $O$. diffusa and $O$. corymbosa. The $(\mathrm{G}+\mathrm{C})$ contents (\%) ranged from $54.27 \%$ to $58.62 \%$ in ITS1 region of $O$. diffusa and ranged from $51.86 \%$ to $62.50 \%$ in $O$. corymbosa (Table 1). Most of ITS2 regions showed higher (G+C) contents (\%) than ITS1 regions, ranged from $60.00 \%$ to $61.22 \%$ in $O$. diffusa and ranged from $58.69 \%$ to $61.60 \%$ in $O$. corymbosa, except for ST-4 and ST-6 populations in O. corymbosa (Table 1). In this study, expectedly, the (G+C) contents (\%) of the 5.8S rRNA region were absolutely identical to $53.37 \%$ among 11 sequences of both species.

Homology analysis of $O$. diffusa and O. corymbosa

Among nine $O$. corymbosa populations and two $O$. diffusa populations, ST-4 population of $O$. corymbosa had the lowest homology of $93.5 \%$ with ST-S population, according to the total ITS region sequence analysis (Table 2). There was a number of nucleotides and length variations in ITS1 region (Fig. 1A, Table 3), but this dissimilarity was mainly owing to the sequence variation of the two populations in ITS2 region, with a low homology of $89.1 \%$ (Table 4).

Table 2 Homology matrix (\%) of 11 total ITS region sequences

\begin{tabular}{|c|c|c|c|c|c|c|c|c|c|c|c|}
\hline Population & LT-L & LT-S & ST-1 & ST-2 & ST-3 & ST-4 & ST-5 & ST- 6 & ST-7 & ST-L & ST-S \\
\hline LT-L & 100 & & & & & & & & & & \\
\hline LT-S & 97.0 & 100 & & & & & & & & & \\
\hline ST-1 & 96.3 & 99.8 & 100 & & & & & & & & \\
\hline ST-2 & 99.2 & 98 & 98.2 & 100 & & & & & & & \\
\hline ST-3 & 94.5 & 97.3 & 97.4 & 96 & 100 & & & & & & \\
\hline ST-4 & 94.0 & 94.0 & 94.0 & 94.2 & 93.9 & 100 & & & & & \\
\hline ST-5 & 97.0 & 100 & 99.8 & 98.0 & 97.4 & 94.0 & 100 & & & & \\
\hline ST-6 & 96.1 & 100 & 99.9 & 98.0 & 97.3 & 94.0 & 100 & 100 & & & \\
\hline ST-7 & 94.9 & 95.7 & 95.9 & 96.0 & 97.6 & 93.9 & 95.9 & 95.8 & 100 & & \\
\hline ST-L & 98.4 & 96.4 & 96.3 & 98.5 & 94.1 & 94.0 & 96.4 & 96.1 & 95.3 & 100 & \\
\hline ST-S & 98.8 & 96.7 & 96.8 & 98.5 & 94.6 & 93.5 & 96.6 & 96.7 & 94.9 & 97.7 & 100 \\
\hline
\end{tabular}


Table 3 Homology matrix (\%) of 11 ITS1 region sequences

\begin{tabular}{|c|c|c|c|c|c|c|c|c|c|c|c|}
\hline Population & LT-L & LT-S & ST-1 & ST-2 & ST-3 & ST-4 & ST-5 & ST-6 & ST-7 & ST-L & ST-S \\
\hline LT-L & 100 & & & & & & & & & & \\
\hline LT-S & 99.7 & 100 & & & & & & & & & \\
\hline ST-1 & 91.5 & 91.5 & 100 & & & & & & & & \\
\hline ST-2 & 99.1 & 99.1 & 91.5 & 100 & & & & & & & \\
\hline ST-3 & 92.0 & 92 & 96.4 & 92.9 & 100 & & & & & & \\
\hline ST-4 & 95.4 & 95.4 & 93.4 & 95.4 & 95.4 & 100 & & & & & \\
\hline ST-5 & 95.7 & 95.7 & 93.7 & 96.5 & 95.1 & 95.4 & 100 & & & & \\
\hline ST-6 & 95.7 & 95.7 & 93.3 & 96.6 & 94.7 & 95.4 & 100 & 100 & & & \\
\hline ST-7 & 74.8 & 73.3 & 100 & 89.6 & 96.4 & 93.4 & 91.7 & 91.3 & 100 & & \\
\hline ST-L & 80.1 & 79.6 & 91.0 & 97.0 & 90.6 & 94.1 & 93.9 & 94 & 72.5 & 100 & \\
\hline ST-S & 95.7 & 95.7 & 93.3 & 96.6 & 94.7 & 95.4 & 100 & 100 & 91.3 & 94 & 100 \\
\hline
\end{tabular}

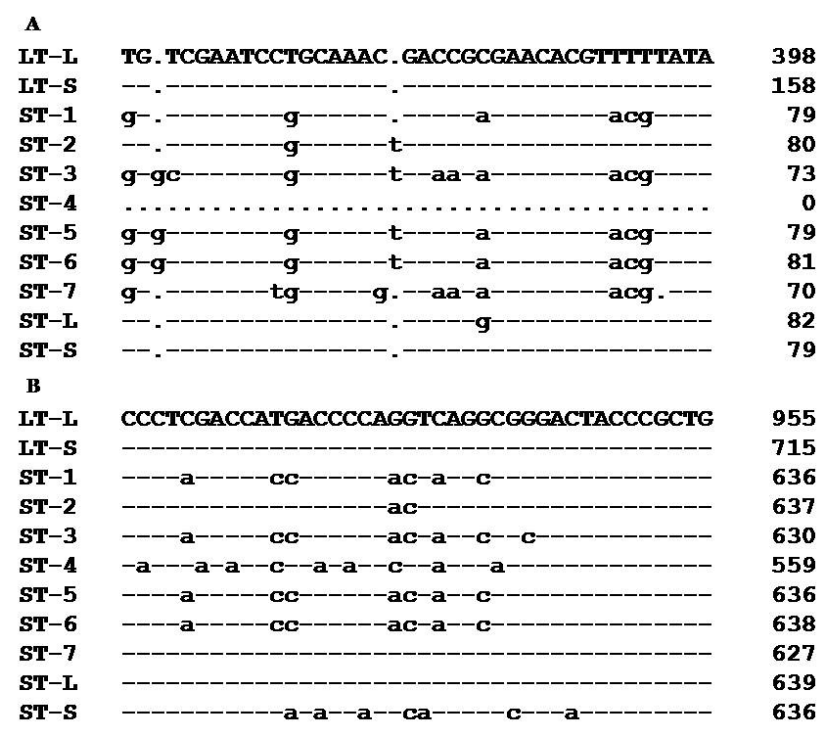

Fig. 1 DNA alignment of $O$. diffusa, $O$. corymbosa using total ITS region. Partial alignment of the total ITS regions showing notable base phlomorphisms among different populations of $O$. diffusa and O. corymbosa in ITS1 (A) and ITS2 (B) regions. The letters on the top line represent the base letters in sequence alignment. Sequences different from the uppermost sequence are marked as their own sequences in lowercase. '-' represents the base being identical to the uppermost sequence. ' ' represents gap

ST-4 population showed relatively low homology with all the other populations of either $O$. corymbosa or $O$. diffusa, except for ST-S population, according to the total ITS region sequence analysis (Table 2). Similar results were obtained from homology compared with the ITS2 region sequence. ST-4 population not only showed relatively low homology with other populations, but also appeared more information sites when aligned with other ITS2 region sequences (Fig. 1B). However, according to the ITS1 region sequence analysis, ST-4 population showed $93.4 \sim 95.4 \%$ identity with other populations, while ST-7 having 100\% homology with ST-1, showed relatively low homology with other populations, especially two populations of $O$. diffusa and ST-L population (Table 3). These results were not congruent with the evidence of $\mathrm{Li}$ et al. (2010) that $O$. diffusa and $O$. corymbosa could be clearly authenticated by either ITS1 or ITS2 region sequence. In the report of $\mathrm{Li}$ et al. (2010), O. diffusa showed its own informative nucleotide sites, different from those of $O$. corymbosa, through which even confused $O$. diffusa or $O$. corymbosa starting materials could be authenticated. In our study, both LT-L and LT-S populations belonged to the same species, $O$. diffusa, however, did not show identical nucleotide sequences in the ITS1 and ITS2 regions (Fig. 1A, B). In addition, ST-1 and ST-7 both belonging to the same species, O. corymbosa, showed $100 \%$ identity in ITS1 region, as well as ST-5, ST-6, and ST-S (Table 3). In the ITS2 region, ST-1, ST-5, ST-6, and ST-S populations of $O$. corymbosa showed $100 \%$ identity with each other (Table 4).

Summarizing the above results, it concluded that the ITS1 and ITS2 region sequences of both $O$. diffusa populations used in this experiment were intra-specifically conserved, while those of $O$. corymbosa populations were not intra-specifically conversed. This suggestion was not supported by the evidence of Guo et al. (2011) that the total ITS region of $O$. diffusa and $O$. corymbosa were intra-specifically conserved but inter-specifically variable, thus, enough to differentiate these both species. In our study, the nucleotide variations appeared in the total ITS regions of different $O$. corymbosa populations, and some populations showed more similar ITS regions with $O$. diffusa populations, e.g. ST-2 had higher homology with LT-T and LT-S compared to other $O$. corymbosa populations. 
Table 4 Homology matrix (\%) of 11 ITS2 region sequences

\begin{tabular}{|c|c|c|c|c|c|c|c|c|c|c|c|}
\hline Population & LT-L & LT-S & ST-1 & ST-2 & ST-3 & ST-4 & ST-5 & ST-6 & ST-7 & ST-L & ST-S \\
\hline LT-L & 100 & & & & & & & & & & \\
\hline LT-S & 100 & 100 & & & & & & & & & \\
\hline ST-1 & 95.6 & 94.3 & 100 & & & & & & & & \\
\hline ST-2 & 98.9 & 98.9 & 98.1 & 100 & & & & & & & \\
\hline ST-3 & 93.4 & 92.1 & 97.8 & 96.2 & 100 & & & & & & \\
\hline ST-4 & 89.8 & 89.8 & 89.1 & 89.5 & 88.7 & 100 & & & & & \\
\hline ST-5 & 96.3 & 96.3 & 100 & 98.1 & 97.8 & 89.1 & 100 & & & & \\
\hline ST-6 & 95.6 & 94.3 & 100 & 98.1 & 97.8 & 89.1 & 100 & 100 & & & \\
\hline ST-7 & 96.7 & 91.7 & 96.1 & 98.1 & 96.8 & 89.8 & 95.9 & 96.1 & 100 & & \\
\hline ST-L & 98.5 & 91.7 & 93.9 & 98.9 & 91.8 & 89.8 & 96.3 & 93.9 & 89.4 & 100 & \\
\hline ST-S & 96.3 & 96.3 & 100 & 98.1 & 97.8 & 89.1 & 100 & 100 & 95.9 & 96.3 & 100 \\
\hline
\end{tabular}

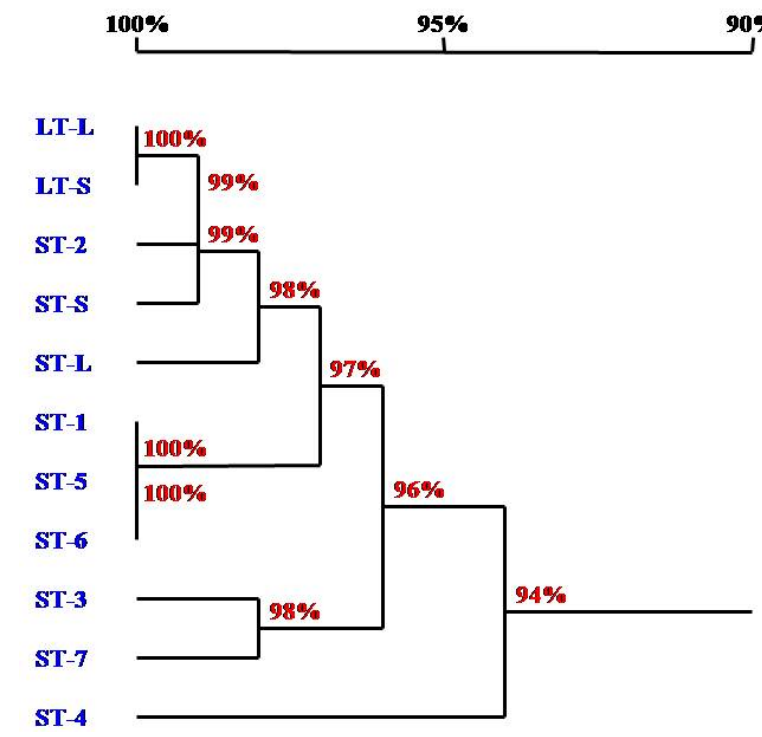

Fig. 2 Phylogenetic tree constructed by total ITS region sequences of nine populations of $O$. corymbosa and two populations of $O$. diffusa

Phylogeny of O.diffusa and O. corymbosa

Based on the phylogenetic tree constructed by 11 total ITS region sequences, $O$. diffusa and $O$. corymbosa had not been anticipated divided into two clades, on the contrary, the LT-L population of $O$. diffusa population formed one monophyletic group with LT-S, near to ST-2 population of O. corymbosa, and ST-S population sharing $99 \%$ similarity (Fig. 2). The ST-4 population of $O$. corymbosa showed the highest dissimilarity with all populations, sharing $94 \%$ similarity according to clustering analysis of the total ITS region sequence. From the phylogenetic tree constructed by ITS1 region sequence, the ST-L population of $O$. corymbosa as the Outgroup shared $89 \%$ similarity with two

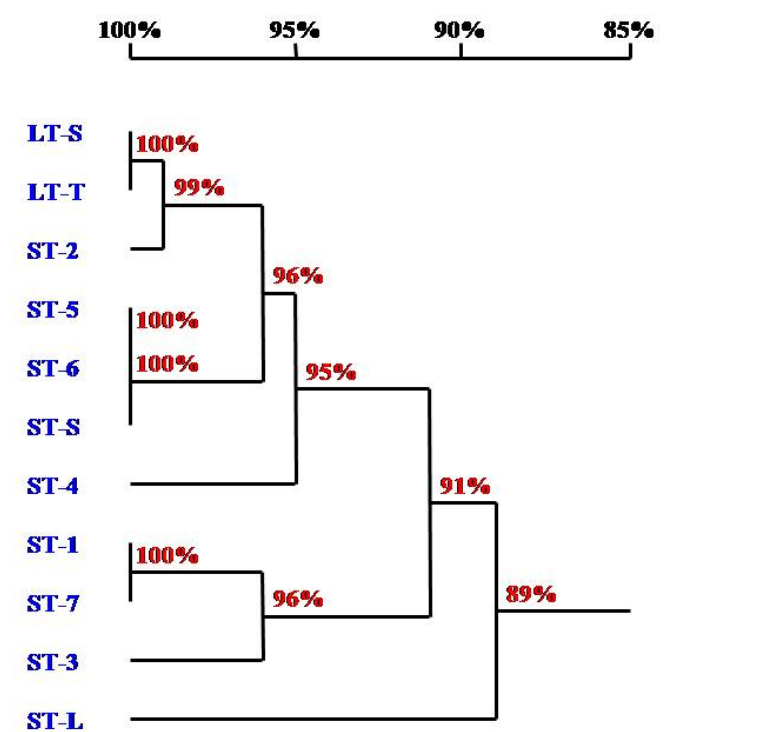

Fig. 3 Phylogenetic tree constructed by ITS1 region sequences of nine populations of $O$. corymbosa and two populations of $O$. diffusa

monophletic groups respectively formed by three $O$. corymbosa populations and the other $O$. corymbosa populations interfused with $O$. diffusa. The group simply formed by $O$. corymbosa populations shared the lowest similarity of $96 \%$, while the other group shared $95 \%$ similarity between ST-4 and other populations (Fig. 3). In the ITS2 region phylogenetic tree, ST-4 population of $O$. corymbosa became the Outgroup, showing $89 \%$ similarity with other populations (Fig. 4). All populations of $O$. diffusa together with populations ST-2 and ST-L of O. corymbosa formed one monophyletic group, showing $96 \%$ identity in the lowest level, while other $O$. corymbosa populations formed another monophyletic group, showing $96 \%$ identity in the lowest level as well (Fig. 4). 


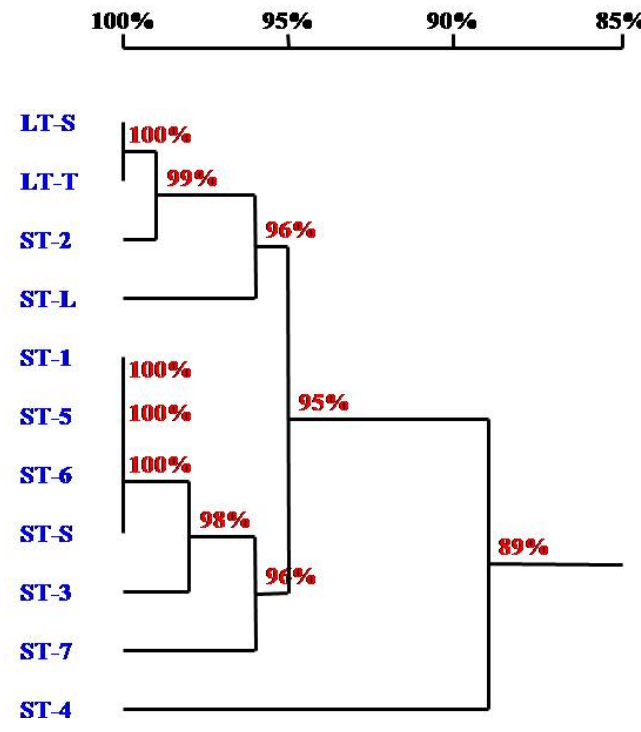

Fig. 4 Phylogenetic tree constructed by ITS2 region sequences of nine populations of $O$. corymbosa and two populations of $O$. diffusa

The clustering analysis on $O$. diffusa and $O$. corymbosa species showed that all species were not form distantly separate clusters, contrarily $O$. diffusa clade interlaced with some $O$. corymbosa populations, formed one multi-level group. This suggesting was not congruent with the evidence of Li et al. (2010) that $O$. diffusa and O. corymbosa could be recognized as distinct species in the genus Oldenlandia phylogenetic tree. The results could be comprehended by the currently available limited sampling of Asian species. To further understand the phylogeny of $O$. diffusa and $O$. corymbosa, a broader concept of samples could be investigated in the recent molecular phylogenetic analysis.

In conclusion, the total ITS sequences of $O$. diffusa and $O$. corymbosa populations collected from Korea were successfully amplified using common primer set, ITS4/ITS5. However, due to currently limited sampling of both species, $O$. diffusa and $O$. corymbosa could not yet be authenticated only based on ITS region sequences in the present study. To ensure proper use of herbal materials for efficient treatment and correct identification of the two medicinal herbs, $O$. diffusa and $O$. corymbosa, additional markers and identification methods should be applied to achieve correct authentication.

\section{Acknowledgements}

This research was supported by Nutraceutical Bio Brain Korea 21 Project Group.

\section{References}

Corbineau F, Côme D (1980) Principaux paramètres de la germination des graines d'Oldenlandia corymbosa L. (Rubiacée tropicale). Physiol Vég 18:259-273

Guo X, Simmons MP, But PPH, Shaw PC, Wang RJ (2011) Application of DNA barcodes in Hedyotis L. (Spermacoceae, Rubiaceae). J Syst Evol 49:203-212. doi:10.1111/j.17596831.2011.00130.x

Hao MG, Liu ZQ, Wang JL (2004) Application of the sequences of rDNA ITS to identify Chinese crude drug Hedyotis diffusa. J Anhui Norm Univ (Nat Sci) 27:188-191

Kang HW, Cho YG, Yoon UH, Eun MY (1998) A rapid DNA extraction method for RFLP and PCR analysis from a single dry seed. Plant Mol Biol Rep 16:1-9

Kårehed J, Groeninckx I, Dessein S, Motley TJ, Bremer B (2008) The phylogenetic utility of chloroplast and nuclear DNA markers and the phylogeny of the Rubiaceae tribe Spermacoceae. Mol Phylogenet Evol 49:843-866. doi:10.1016/j. ympev.2008.09.025

Kitani Y, Shu Z, Batkhuu J, Sanchir C, Komatsu K (2011) Genetic diversity of Ephedra plants in Mongolia inferred from internal transcribed spacer sequence of nuclear ribosomal DNA. Biol Pharm Bull 34: 717-726

Kitani Y, Shu Z, Batkhuu J, Sanchir C, Komatsu K (2011) Genetic diversity of Ephedra plants in Mongolia inferred from internal transcribed spacer sequence of nuclear ribosomal DNA. Biol Pharm Bull 34:717-726

Kitani Y, Zhu S, Omote T, Tanaka K, Batkhuu J, Sanchir C, Fushimi H, Mikage M, Komatsu K (2009) Molecular analysis and chemical evaluation of Ephedra plants in Mongolia. Biol Pharm Bull 32:1235-1243

Ko WC (1999) Hedyotis. In: Luo XR (ed) Flora Republicae Popularis Sinicae. Science Press, Beijing, vol 71, pp 32-77

Lau P, Peng Y, Zhao Z (2004) Microscopic identification of Chinese patent medicine (1) - Wu Zi Yan Zong Wan. Nat Med 58:258-265

Li M, Jiang RW, Hon PM, Cheng L, Li LL, Zhou JR, Shaw PC, But PP (2010) Authentication of the anti-tumor herb Baihuasheshecao with bioactive marker compounds and molecular sequences. Food Chem 119:1239-1245. doi:10.1016/j.foodchem. 2009.09.113

Liang ZT, He MF, Fong WF, Jiang ZH, Zhao ZZ (2008) A comparable, chemical and pharmacological analysis of the traditional Chinese medicinal herbs Oldenlandia diffusa and $O$. corymbosa and a new valuation of their biological potential. Phytomedicine 15:259-267. doi:10.1016/l.phymed.2008.01. 003

Liang ZT, Jiang ZH, Leung KSY, Peng Y, Zhao ZZ (2006) Distinguishing the medicinal herb Oldenlandia diffusa from similar species of the same genus using fluorescence microscopy. Microsc Res Tech 69:277-282. doi:10.1002/jemt.20312

Lin CC, Chen FY, Namba T (1987) Development of crude drug resources from Taiwan: pharmacognostical studies on a Chinese crude drug. Shoyakugaku Zasshi 41:180-188 
Liu ZQ, Hao MG (2005) Determination of rDNA ITS sequences in Hedyotis diffusa. Shaanxi J Tradl Chin Med 26:167-169

Liu ZQ, Hao MG, Wang JL (2004) Application of allele-specific primer in the identification of Hedyotis diffusa. Zhong Yao Cai 27:484-487

Long CF, Kakiuchi N, Takahashi A, Komatsu K, Cai SQ, Makage M (2004) phylogenetic analysis of the DNA sequence of the non-coding region of nuclear ribosomal DNA and chloroplast of Ephedra plants in China. Planta Med 70:1080-1084. Doi:10.1055/s-2004-832651

Shan BE, Zhang JY, Du XN (2001) Immunomodulatory activity and anti-tumor activity of Oldenlandia diffusa in vitro. Zhongguo Zhong Xi Yi Jie He Za Zhi 21:370-374

The State Pharmacopoeia Committee of China (2005) The appendix of Chinese pharmacopoeia. The Chemical Industry Press, Beijing, vol I, pp 22
White TJ, Bruns T, Lee S, Taylor J (1990) Amplification and direct sequencing of fungal ribosomal RNA genes for phylogenetics. In: Innis MA, Gelfand DH, Sninsky JJ, White TJ (eds) PCR protocols-a guide to methods and applications. Academic Press, San Diego, Calif, pp 315-322

Wu PK, Tai WCS, Liang ZT, Zhao ZZ, Hsiao WLW (2009) Oleanolic acid isolated from Oldenlandia diffusa exhibits a unique growth inhibitory effect against ras-transformed fibroblasts. Life Sci 85:113-121. Doi:10.1016/j.lfs.2009.04. 025

Xu GJ, Xu LS, Wang ZT (1997) Species systematization and quality evaluation of commonly used Chinese traditional drugs. South-China Edition, Fujian: Fujian Science and Technology Publisher, Fuzhou, China, vol 3-4

Zhao ZZ, Li YS (2007) Easily confused Chinese medicines in Hong Kong. Hong Kong: Chinese Medicine Merchants Association Ltd., Hong Kong 